\title{
Optimal On-Line Colorings for Minimizing the Number of ADMs in Optical Networks
}

\author{
(Extended Abstract)
}

\author{
Mordechai Shalom ${ }^{1,3}$, Prudence W.H. Wong ${ }^{2}$, and Shmuel Zaks ${ }^{1, \star}$ \\ ${ }^{1}$ Department of Computer Science, Technion, Haifa, Israel \\ \{cmshalom, zaks\}@cs.technion.ac.il \\ ${ }^{2}$ Department of Computer Science, The University of Liverpool, Liverpool, UK \\ pwong@csc.liv.ac.uk \\ 3 Tel-Hai Academic College, Upper Galilee, 12210, Israel \\ cmshalom@telhai.ac.il
}

\begin{abstract}
We consider the problem of minimizing the number of ADMs in optical networks. All previous theoretical studies of this problem dealt with the off-line case, where all the lightpaths are given in advance. In a real-life situation, the requests (lightpaths) arrive at the network on-line, and we have to assign them wavelengths so as to minimize the switching cost. This study is thus of great importance in the theory of optical networks. We present an on-line algorithm for the problem, and show its competitive ratio to be $\frac{7}{4}$. We show that this result is best possible in general. Moreover, we show that even for the ring topology network there is no on-line algorithm with competitive ratio better than $\frac{7}{4}$. We show that on path topology the competitive ratio of the algorithm is $\frac{3}{2}$. This is optimal for this topology. The lower bound on ring topology does not hold when the ring is of bounded size. We analyze the triangle topology and show a tight bound of $\frac{5}{3}$ for it. The analyzes of the upper bounds, as well as those for the lower bounds, are all using a variety of proof techniques, which are of interest by their own, and which might prove helpful in future research on the topic.
\end{abstract}

\section{Introduction}

\subsection{Background}

Optical wavelength-division multiplexing (WDM) is today the most promising technology that enables us to deal with the enormous growth of traffic in communication networks, like the Internet. A communication between a pair of nodes is done via a lightpath, which is assigned a certain wavelength. In graph-theoretic terms, a lightpath is a simple path in the network, with a color assigned to it.

Given a WDM network $G=(V, E)$ comprising optical nodes and a set of fullduplex lightpaths $P=\left\{p_{1}, p_{2}, \ldots, p_{N}\right\}$ of $G$, the wavelength assignment (WLA)

\footnotetext{
* This research was partly supported by the EU Project "Graphs and Algorithms in Communication Networks (GRAAL)" - COST Action TIST 293.
} 
task is to assign a wavelength to each lightpath $p_{i}$. Most of the studies in optical networks dealt with the issue of assigning colors to lightpaths, so that every two lightpaths that share an edge get different colors.

When the various parameters comprising the switching mechanism in these networks became clearer, the focus of studies shifted, and today a large portion of the studies concentrates on the total hardware cost. The key point here is that each lightpath uses two Add-Drop Multiplexers (ADMs), one at each endpoint. If two adjacent lightpaths, i.e. lightpaths sharing a common endpoint, are assigned the same wavelength, then they can use the same ADM. Because ADMs are designed to be used mainly in ring and path networks in which the degree af a node is at most two, an ADM may be shared by at most two lightpaths. The total cost considered is the total number of ADMs. A more detailed technical explanation can be found in GLS98.

Lightpaths sharing ADMs in a common endpoint can be thought as concatenated, so that they form longer paths or cycles. These paths/cycles do not use any edge $e \in E$ twice, for otherwise they cannot use the same wavelength which is a necessary condition to share ADMs.

\subsection{Previous Work}

Minimizing the number of ADMs in optical networks is a main research topic in recent studies. The problem was introduced in GLS98 for the ring topology. An approximation algorithm for ring topology with approximation ratio of $3 / 2$ was presented in CW02, and was improved in SZ04, EL04 to 10/7 $+\epsilon$ and $10 / 7$, respectively. For general topology EMZ02 describe an algorithm with approximation ratio of $8 / 5$. The same problem was studied in CFW02 and an algorithm with an approximation ratio of $\frac{3}{2}+\epsilon$ was presented. This algorithm is further analyzed in FSZ06b.

The problem of on-line path coloring is studied in earlier works, such as [LV98]. The problem studied in these works has a different objective function, namely the number of colors.

All previous theoretical studies on the problem of minimizing the number of switches dealt with the off-line case, where all the lightpaths are given in advance. In a real-life situation, the requests (lightpaths) arrive at the network on-line, and we have to assign them wavelengths so as to minimize the switching cost. An on-line algorithm is said to be $c$-competitive if for any sequence of lightpaths, the number of ADMs used is at most $c$ times that used by the optimal offline algorithm (see BEY98).

\subsection{Our Contribution}

We present an on-line algorithm with competitive ratio of $\frac{7}{4}$ for any network topology. We prove that no algorithm has a competitive ratio better than $\frac{7}{4}$ even if the topology is a ring.

We show that the same algorithm has a competitive ratio of $\frac{3}{2}$ in path topologies, and that this is also a lower bound for on-line algorithms in this topology. 
The lower bound on ring topology does not hold when the ring is of a bounded size. We study the triangle topology, and show a tight bound of $\frac{5}{3}$ for the competitive ratio on this topology, using another algorithm.

The analyses of the upper bounds, as well as those for the lower bounds, use a variety of proof techniques, which are of interest on their own, and which might prove helpful in future research on the topic.

In Section 2 we describe the problem and some preliminary results. The algorithm and its competitive analysis are presented in Section 3 In Section 4 we present lower bounds for the competitive ratio of the problem on general topology, ring and path topologies. In Section 5 we present tight bounds for triangle networks. We conclude with discussion and open problems in Section 6 Some proofs are sketched in this Extended Abstract; for full details the reader is referred to SWZ07.

\section{Preliminaries}

An instance $\alpha$ of the problem is a pair $\alpha=(G, P)$ where $G=(V, E)$ is an undirected graph and $P$ is a set of simple paths in $G$. In an on-line instance, the graph $G$ is known in advance and the set $P$ of paths is given on-line. In this case we denote $P=\left\{p_{1}, p_{2}, \ldots, p_{N}\right\}$ where $p_{i}$ is the $i$-th path of the input and $P_{i}=\left\{p_{j} \in P \mid j \leq i\right\}$ consists of the first $i$ paths of the input.

In this work we need a number of notions introduced in FSZ06a.

- The paths $p, p^{\prime} \in P$ are conflicting or overlapping if they have an edge in common. This is denoted as $p \asymp p^{\prime}$. The graph of the relation $\asymp$ is called the conflict graph of $(G, P)$.

- A proper coloring (or wavelength assignment) of $P$ is a function $w: P \mapsto \mathbb{N}$, such that $w(p) \neq w\left(p^{\prime}\right)$ whenever $p \asymp p^{\prime}$.

- A valid chain (resp. cycle) of $\alpha=(G, P)$ is a path (resp.cycle) formed by the concatenation of distinct paths $p_{i_{0}}, p_{i_{1}}, \ldots, p_{i_{k-1}} \in P$ that do not go over the same edge twice. Note that the paths of a valid chain (resp. cycle) constitute an independent set of the conflict graph.

- A solution $S$ of an instance $\alpha=(G, P)$ is a set of valid chains and valid cycles of $P$ such that each $p \in P$ appears in exactly one of these sets.

Note that $w$ is a proper coloring if and only if for any color $c \in \mathbb{N}, w^{-1}(c)$ is an independent set in the conflict graph.

In the sequel we introduce the shareability graph, which together with the conflict graph constitutes another (dual) representation of the instance $\alpha$. In the sequel, except one exception, we will use the dual representation of the problem.

- The shareability graph of an instance $\alpha=(G, P)$, is the edge-labelled multigraph $\mathcal{G}_{\alpha}=\left(P, E_{\alpha}\right)$ such that there is an edge $e=(p, q)$ labelled $u$ in $E_{\alpha}$ if and only if $p \nprec q$, and $u$ is a common endpoint of $p$ and $q$ in $G$.

- A valid chain (resp. cycle) of $\mathcal{G}_{\alpha}$ is a simple path $p_{i_{0}}, p_{i_{1}}, \ldots, p_{i_{k-1}}$ of $\mathcal{G}_{\alpha}$, such that any two consecutive edges in the path (resp. cycle) have distinct labels 
and its node set is properly colorable with one color (in $G$ ), or in other words constitutes an independent set of the conflict graph.

- The sharing graph of a solution $S$ of an instance $\alpha=(G, P)$, is the following subgraph $\mathcal{G}_{\alpha, S}=\left(P, E_{S}\right)$ of $\mathcal{G}_{\alpha}$. Two lightpaths $p, q \in P$ are connected with an edge labelled $u$ in $E_{S}$ if and only if they are consecutive in a chain or cycle in the solution $S$, and their common endpoint is $u \in V$. We will usually omit the index $\alpha$ and simply write $\mathcal{G}_{S} . d(p)$ is the degree of node $p$ in $\mathcal{G}_{S}$.

Example: Let $\alpha=(G, P)$ be the instance in the left side of Figure 1. Its shareability graph $\mathcal{G}_{\alpha}$ is the graph at midle. In this instance $P=\{a, b, c, d\}$, and it constitutes the set of nodes of $\mathcal{G}_{\alpha}$. The edges together with their labels are $E_{\alpha}=$ $\{(b, c, u),(a, c, w),(a, b, x),(a, d, x)\}$, because $a$ and $b$ can be joined in their common endpoint $x$, etc.. Note that, for instance $(b, d, x) \notin E_{\alpha}$, because although $b$ and $d$ share a common endpoint $x$, they can not be concatenated, because they have the edge $(x, u)$ in common. The corresponding conflict graph is at the right side of the figure. It has the same node set and one edge, namely $(b, d)$. The paths $b, d \in P$ are conflicting because they have a common edge, i.e. $(u, x)$.
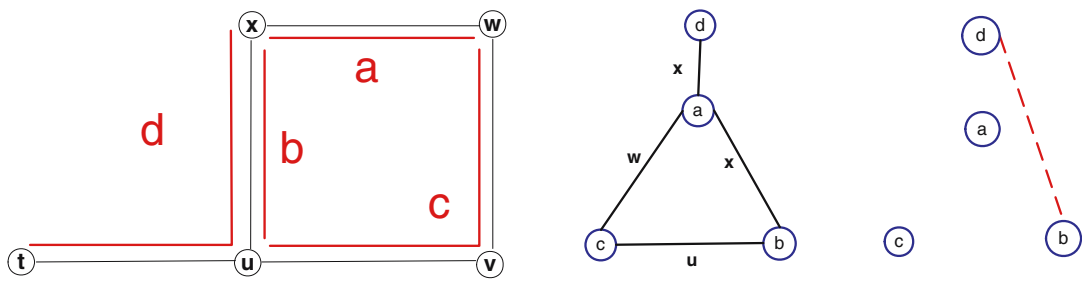

Fig. 1. A sample input

Note that the edges of the conflict graph are not in $E_{\alpha}$. This immediately follows from the definitions. Note also that, for any node $v$ of $\mathcal{G}_{\alpha}$, the set of labels of the edges adjacent to $v$ is of size at most two.

Valid chains and cycles of $\mathcal{G}_{\alpha}$ correspond to valid chains and cycles of the instance $\alpha$. In the above example the chain $a, d$ which is the concatenation of the paths $a$ and $d$ in the graph $G$, corresponds to the simple path $a, d$ in $\mathcal{G}_{\alpha}$ and the cycle $a, b, c$ which is a cycle formed by the concatenation of three paths in $G$ corresponds to the cycle $a, b, c$ in $\mathcal{G}_{\alpha}$. Note that no two consecutive labels are equal in this cycle. On the other hand the paths $b, a, d$ can not be concatenated to form a chain, because this would require the connection of $a$ to both $b$ and $d$ at node $x$. The corresponding path $b, a, d$ in $\mathcal{G}_{\alpha}$ is not a chain because the edges $(b, a)$ and $(a, d)$ have the same label, namely $x$.

$S=\{(d, a, c),(b)\}$ is a solution with two chains. The sharing graph of this solution has two edges $(d, a)$ and $(a, c)$. Note that for a chain of size at most two, the distinct labelling condition is satisfied vacuously, and the independent set condition is satisfied because no edge of $\mathcal{G}_{\alpha}$ can be an edge of the conflict graph.

We define $\forall i \in\{0,1,2\}, D_{i}(S) \stackrel{\text { def }}{=}\{p \in P \mid d(p)=i\}$ and $d_{i}(S) \stackrel{\text { def }}{=}\left|D_{i}(S)\right|$. 
Note that $d_{0}(S)+d_{1}(S)+d_{2}(S)=|P|=N$.

An edge $(p, q) \in E_{S}$ with label $u$ corresponds to a concatenation of two paths with the same color at their common endpoint $u$. Therefore these two endpoints can share an ADM operating at node $u$, thus saving one ADM. We conclude that every edge of $E_{S}$ corresponds to a saving of one ADM. When no ADMs are shared, each path needs two ADM's, a total of $2 N$ ADMs. Therefore the cost of a solution $S$ is

$$
\operatorname{cost}(S)=2|P|-\left|E_{S}\right|=2 N-\left|E_{S}\right| \text {. }
$$

The objective is to find a solution $S$ such that $\operatorname{cost}(S)$ is minimum, in other words $\left|E_{S}\right|$ is maximum.

The following definitions and Lemma appeared in [FSZ06b, we repeat them here for completeness.

Given a solution $S, d(p) \leq 2$ for every node $p \in P$. Therefore, the connected components of $\mathcal{G}_{S}$ are either paths or cycles. Note that an isolated vertex is a special case of a path. Let $\mathcal{P}_{S}$ be the set of the connected components of $\mathcal{G}_{S}$ that are paths. Clearly, $\left|E_{S}\right|=N-\left|\mathcal{P}_{S}\right|$. Therefore $\operatorname{cost}(S)=2 N-\left|E_{S}\right|=N+\left|\mathcal{P}_{S}\right|$.

Let $S^{*}$ be a solution with minimum cost. For any solution $S$ we define

$$
\epsilon(S) \stackrel{\text { def }}{=} \frac{d_{0}(S)-d_{2}(S)-2\left|\mathcal{P}_{S^{*}}\right|}{N}
$$

Lemma 2.1 For any solution $S, \operatorname{cost}(S)=\operatorname{cost}\left(S^{*}\right)+\frac{1}{2} N(1+\epsilon(S))$.

Proof. Clearly $\left|E_{S^{*}}\right|=N-\left|\mathcal{P}_{S^{*}}\right|$. On the other hand $2\left|E_{S}\right|$ is the sum of the degrees of the nodes in $\mathcal{G}_{S}$, namely $2\left|E_{S}\right|=d_{1}(S)+2 d_{2}(S)=N-d_{0}(S)+d_{2}(S)$. We conclude:

$$
\begin{aligned}
\operatorname{cost}(S) & -\operatorname{cost}\left(S^{*}\right)=\left|E_{S^{*}}\right|-\left|E_{S}\right|=N-\left|\mathcal{P}_{S^{*}}\right|-\frac{N-d_{0}(S)+d_{2}(S)}{2} \\
& =\frac{N}{2}+\frac{d_{0}(S)-d_{2}(S)-2\left|\mathcal{P}_{S^{*}}\right|}{2}=\frac{1}{2} N\left(1+\frac{d_{0}(S)-d_{2}(S)-2\left|\mathcal{P}_{S^{*}}\right|}{N}\right)
\end{aligned}
$$

\section{Upper Bounds}

In this section we first describe an on-line algorithm, show that it is $7 / 4$-competitive on any network topology and 3/2-competitive on path topology.

\subsection{Algorithm ONLINE-MINADM}

In a general network, when the lightpaths are given one-by-one, we adopt a simple coloring procedure. Basically, a new lightpath with endpoints $u$ and $v$ looks for free ADM at its endpoints. If there are two of the same color, then it first tries to make a cycle with the existing lightpaths, and if this is impossible then it makes a path. If there are free ADMs (at one endpoint, or at both endpoints but of different colors), then it tries to connect to any of them. Otherwise - when there is no free ADM - it is assigned a new color. 
When we attempt to color some lightpath $p_{i}$, a color $\lambda$ is said to be feasible for $p_{i}$, if there is no other lightpath with the same color and overlapping with $p_{i}$. In other words $\lambda$ is feasible for $p_{i}$, if we can assign $w\left(p_{i}\right)=\lambda$ and $w$ is a proper coloring for $P_{i}$.

When a lightpath $p_{i}$ with endpoints $u_{i}$ and $v_{i}$ arrives,

- If there exists a chain of lightpaths with the same color $\lambda$ with endpoints $u_{i}, v_{i}$ and $\lambda$ is feasible for $p_{i}$ then, assign $w\left(p_{i}\right)=\lambda$.

- Otherwise, If there exists a chain of lightpaths with the same color $\lambda$ with one endpoint from $\left\{u_{i}, v_{i}\right\}$ and $\lambda$ is feasible for $p_{i}$ then, assign $w\left(p_{i}\right)=\lambda$.

- Otherwise, assign $w\left(p_{i}\right)=\lambda^{\prime}$, where $\lambda^{\prime}$ is an unused color.

Note that, as in the last clause the algorithm resorts to an unused color, it will never construct two chains with the same color. Therefore in the first clause, the algorithm necessarily closes a cycle.

Algorithm ONLINE-MINADM is obviously correct: $w$ is a proper coloring for $P_{i}$, because if $p_{i}$ is colored by one the first two cases, then it is checked by the algorithm for feasibility, otherwise $w\left(p_{i}\right)$ is assigned an unused color, therefore no other path, in particular no path $p_{j}$ conflicting with $p_{i}$ may have $w\left(p_{j}\right)=w\left(p_{i}\right)$.

In this and the following section we prove the following theorem.

Theorem 3.1 Algorithm ONLINE-MINADM is optimal for

- general topology, with competitive ratio of $\frac{7}{4}$,

- ring topology, with competitive ratio of $\frac{7}{4}$,

- path topology, with competitive ratio of $\frac{3}{2}$.

\subsection{Analysis for General Topology}

Lemma 3.1 The competitive ratio of ONLINE-MINADM is at least $\frac{7}{4}$.

Proof. Let $G$ be a cycle of three nodes $V=\left\{v_{1}, v_{2}, v_{3}\right\}, E=\left\{e_{1}, e_{2}, e_{3}\right\}$ where $e_{1}=\left(v_{1}, v_{2}\right), e_{2}=\left(v_{2}, v_{3}\right), e_{3}=\left(v_{3}, v_{1}\right)$ and let $P=\left\{p_{1}, p_{2}, p_{3}, p_{4}\right\}$ where $p_{1}=$ $\left(e_{3}\right), p_{2}=\left(e_{1}\right), p_{3}=\left(e_{2}, e_{3}\right), p_{4}=\left(e_{1}, e_{2}\right)$. The optimal solution assigns $w\left(p_{1}\right)=$ $w\left(p_{4}\right)=\lambda_{1}$ and $w\left(p_{2}\right)=w\left(p_{3}\right)=\lambda_{2}$, and uses 4 ADMs. Recall that ONLINEMINADM receives the paths of the input one at a time. It assigns $w\left(p_{1}\right)=\lambda_{1}$, then $w\left(p_{2}\right)=\lambda_{1}$ because $\lambda_{1}$ is feasible for $p_{2}$, then $w\left(p_{3}\right)=\lambda_{2}$ because $\lambda_{1}$ is not feasible for $p_{3}$ and finally $w\left(p_{4}\right)=\lambda_{3}$, because neither $\lambda_{1}$ nor $\lambda_{2}$ are feasible for $p_{4}$. It uses 7 ADM's in total.

In the sequel $S$ is a solution returned by the ONLINE-MINADM and $S^{*}$ is an optimal solution.

Lemma 3.2 The competitive ratio of ONLINE-MINADM is at most $\frac{7}{4}$.

Proof. We direct each edge of $\mathcal{G}_{S^{*}}$, such that each path becomes a directed path and each cycle becomes a directed cycle. The direction chosen for every path (resp. cycle) is arbitrary. Let $\overrightarrow{\mathcal{G}}_{S *}$ be the digraph obtained by this process. 
Unless otherwise stated, $d_{i n}(p)$ and $d_{\text {out }}(p)$ denote the in and out degrees of $p$ in $\overrightarrow{\mathcal{G}}_{S^{*}}$, respectively. Clearly, $\forall p \in P, d_{i n}(p) \leq 1$ and $d_{\text {out }}(p) \leq 1$. The following definitions refer to $\overrightarrow{\mathcal{G}}_{S *}$ :

$L A S T^{*}$ is the set of nodes that do not have successors in $\overrightarrow{\mathcal{G}}_{S^{*}}$, namely

$$
L A S T^{*} \stackrel{\text { def }}{=}\left\{p \in P \mid d_{\text {out }}(p)=0\right\} .
$$

Note that $\left|L A S T^{*}\right|=\left|P_{S^{*}}\right|$.

The functions Next* and Prev* are defined as expected: Next* (resp. Prev ${ }^{*}$ ) maps a node $p$ to the next (resp. previous) node in $\overrightarrow{\mathcal{G}}_{S^{*}}$ whenever such a node exists, namely:

$$
\text { Next }^{*}: P \backslash L A S T^{*} \mapsto P
$$

and $\operatorname{Next}^{*}(p)$ is the unique node $u$ such that there is an edge from $p$ to $u$ in $\overrightarrow{\mathcal{G}}_{S^{*}}$. Prev $^{*}=$ Next ${ }^{*-1}$.

With these definitions in hand, we partition $D_{0}(S)$ into the sets $A, B, C$ and $D$ using the following classification procedure : Given a path $p \in D_{0}(S)$, if $p \in$ $L A S T^{*}$ then $p$ is in $A$ and $f_{A}(p)=p$. Otherwise, there is a node $q=\operatorname{Next}^{*}(p)$, we decide according to the degree of $q$ in $S$ : if it has degree 2 , then $p$ is in $B$ and $f_{B}(p)=q$, if it has degree 1 , then $p$ is in $C$ and $f_{C}(p)=\{p, q\}$, otherwise $q$ has degree 0 , then $p$ is in $D$.

It is also immediate from the description that $f_{A}: A \mapsto L A S T^{*}, f_{B}: B \mapsto$ $D_{2}(S)$ and $f_{C}: C \mapsto 2^{P}$.

We first show that $D=\emptyset$. Assume, by contradiction that $p \in D$ for some $p \in$ $D_{0}(S)$. Then there is $q \in D_{0}(S)$ such that $q=N e x t^{*}(p)$, therefore $(p, q) \in E_{S^{*}} \subseteq$ $E_{\alpha}$. ONLINE-MINADM assigned unique colors to each of $p$ and $q$. Assume without loss of generality that $q$ comes later than $p$ in the input sequence. $p$ is assigned a unique color, therefore it is the only element in its chain. Then $w(p)$ is feasible for $q$. Then the algorithm should assign $w(q)=w(p)$, a contradiction.

$f_{A}(p)=p$, therefore it is a one-to-one function, i.e. $|A| \leq\left|L A S T^{*}\right|=\left|\mathcal{P}_{S^{*}}\right|$.

$f_{B}(p)=N e x t^{*}(p)$. Next* is one-to-one, therefore $f_{B}$ is one-to-one, i.e. $|B| \leq$ $\left|D_{2}(S)\right|=d_{2}(S)$.

We will now show that the sets $f_{C}(p)$ are disjoint. Note that $f_{C}(p)=\{p, q\}$ where $p \in D_{0}(S)$ and $q \notin D_{0}(S)$. Assume that $f_{C}(p) \cap f_{C}\left(p^{\prime}\right) \neq \emptyset$. Let $f_{C}(p)=$ $\{p, q\}$ and $f_{C}\left(p^{\prime}\right)=\left\{p^{\prime}, q^{\prime}\right\}$. Then either $p=p^{\prime}$ or $q=q^{\prime}$. In the latter case $q=\operatorname{Next}^{*}(p)=\operatorname{Next}^{*}\left(p^{\prime}\right)=q^{\prime}$, then $p=p^{\prime}$. In both cases, we have $p=p^{\prime}$. We conclude that if $p \neq p^{\prime}, f_{C}(p) \cap f_{C}(p)=\emptyset$. As the sets $f_{C}(p)$, have exactly 2 elements, we conclude that $|C| \leq \frac{N}{2}$.

We have $d_{0}(S)=\left|D_{0}(S)\right|=|A|+|B|+|C|+|D| \leq\left|\mathcal{P}_{S^{*}}\right|+d_{2}(S)+\frac{N}{2}$. Then

$$
\epsilon(S) \stackrel{\text { def }}{=} \frac{d_{0}(S)-d_{2}(S)-2\left|\mathcal{P}_{S^{*}}\right|}{N} \leq \frac{1}{2}
$$

Substituting this in Lemma 2.1 and recalling that $\operatorname{cost}\left(S^{*}\right) \geq N$ we get

$$
\operatorname{Cost}(S) \leq \operatorname{Cost}\left(S^{*}\right)+\frac{1}{2} N\left(1+\frac{1}{2}\right)=\operatorname{Cost}\left(S^{*}\right)+\frac{3}{4} N \leq \frac{7}{4} \operatorname{Cost}\left(S^{*}\right) .
$$




\subsection{Analysis for Path Topology}

\section{Lemma 3.3 ONLINE-MINADM is $\frac{3}{2}$-competitive in path topology.}

Proof. Let $V=\left\{v_{1}, v_{2}, \ldots\right\}$ be the nodes of the path from left to right, and $\sigma_{i}$ (resp. $\tau_{i}$ ) be the set of paths having $v_{i}$ as their right (resp. left) endpoint. It is well known that the number of ADMs used by an optimal solution is $\Sigma_{i} \max \left\{\left|\sigma_{i}\right|,\left|\tau_{i}\right|\right\}$. In an optimal solution, at each node $v_{i}$, exactly $\min \left\{\left|\sigma_{i}\right|,\left|\tau_{i}\right|\right\}$ pairs of paths are assigned one color per pair. In fact these pairs constitute a maximum matching $M M_{i}$ of the complete bipartite graph $\left(\sigma_{i}, \tau_{i}, \sigma_{i} \times \tau_{i}\right)$. The solution saves $\left|M M_{i}\right|=\min \left\{\left|\sigma_{i}\right|,\left|\tau_{i}\right|\right\}$ ADMs at node $v_{i}$, in other words $E_{S^{*}}=\uplus_{i} M M_{i}$. Note that every matching of a complete bipartite graph can be augmented to a maximum matching. Let $S^{*}$ be an optimal solution, such that the matching in each node is obtained by augmenting the matching done by $S$ to a maximum matching, i.e. $E_{S} \subseteq E_{S^{*}}$.

We will now define a function $f:\left(E_{S^{*}} \backslash E_{S}\right) \mapsto E_{S}$.

Let $e=\left(p_{i}, p_{j}\right) \in E_{S^{*}} \backslash E_{S}$. $e \in E_{S^{*}}=\uplus_{i} M M_{i}$. Let $e \in M M_{k}$. Assume without loss of generality that $i<j$, i.e. path $p_{i}$ appears before $p_{j}$ in the input. As $e \notin E_{S}$, none of $p_{i}, p_{j}$ are paired with any path at node $v_{k}$. Therefore when $p_{j}$ appears in the input $w\left(p_{i}\right)$ is feasible for $p_{j}$, if it is not assigned color $w\left(p_{i}\right)$, this can be only because it is assigned color $w\left(p_{j}\right)=w\left(p_{i^{\prime}}\right)$, for some $i^{\prime}<j$. Let the common node of $p_{j}$ and $p_{i^{\prime}}$ be $v_{k^{\prime}}$. Then $e^{\prime}=\left(p_{j}, p_{i^{\prime}}\right) \in E_{S^{*}}$. We define $f(e)=e^{\prime}$. Note that $e^{\prime}$ is defined uniquely because there can not be a third path except $p_{j}$ and $p_{i^{\prime}}$ getting the same color and ending at node $v_{k^{\prime}}$. Necessarily $k^{\prime} \neq k$, because we know that $p_{j}$ is not paired at node $v_{k}$.

We claim that $f$ is one-to-one. Assume, by contradiction that there is some $e^{\prime \prime} \neq e$, such that $f\left(e^{\prime \prime}\right)=e^{\prime}$. Then $e^{\prime \prime} \in E_{S^{*}}$, therefore $e^{\prime \prime} \in M M_{k^{\prime \prime}}$ for some node $v_{k^{\prime \prime}}$. By the construction of $f, k^{\prime \prime}$ is the other endpoint of $p_{i^{\prime}}$. Let $e^{\prime \prime}=\left(p_{i^{\prime}}, p_{i^{\prime \prime}}\right)$. By the discussion in the previous paragraph, symmetrically it follows that $j<i^{\prime}$, a contradiction. Therefore $f$ is one-to-one, i.e. $\left|E_{S^{*}}\right|-\left|E_{S}\right|=\left|E_{S^{*}} \backslash E_{S}\right| \leq\left|E_{S}\right|$, thus $\left|E_{S}\right| \geq \frac{1}{2}\left|E_{S^{*}}\right|$.

We conclude as follows. $\operatorname{Cost}(S)-\operatorname{Cost}\left(S^{*}\right)=\left|E_{S^{*}}\right|-\left|E_{S}\right| \leq \frac{\left|E_{S^{*}}\right|}{2} \leq \frac{N}{2} \leq$ $\frac{\operatorname{Cost}\left(S^{*}\right)}{2}$, therefore:

$$
\operatorname{Cost}(S) \leq \frac{3}{2} \operatorname{Cost}\left(S^{*}\right)
$$

\section{Lower Bounds}

\subsection{General Topology}

Lemma 4.1 There is no deterministic on-line algorithm with competitive ratio $<\frac{7}{4}$.

Proof. Assume ALG is a deterministic on-line algorithm, with competitive ratio $\rho$. We show that $\rho \geq \frac{7}{4}$. For colors we use numbers $1,2, \ldots$. The color assigned to a lightpath $a$ by ALG is denoted by $w(a)$. We use the network depicted in 

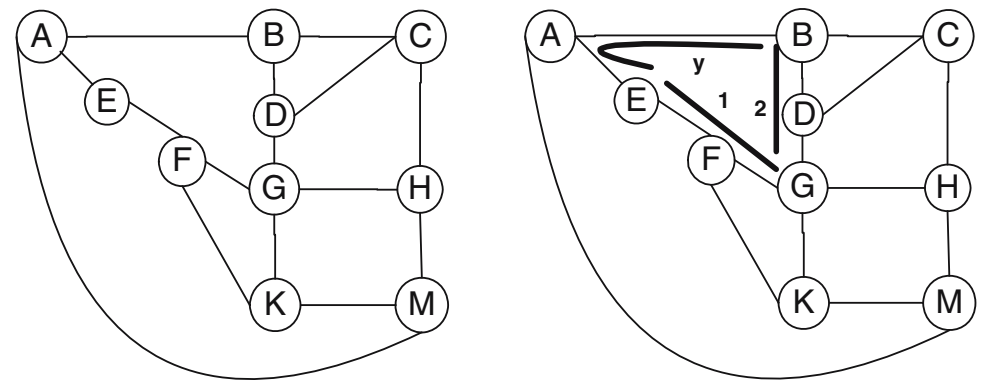

Fig. 2. Proof of Lemma 4.1

Figure 2. The first lightpath in the input is EFG. Without loss of generality, assume $w(E F G)=1$.

The second lightpath in the input is. First assume $w(B D G)=1$. In this case if lightpath EABDG arrives, we have $w(E A B D G)=2$, then when lightpath GFEAB arrives we have $w(G F E A B)=3$. ALG thus uses 7 ADMs, while it is easy to see the an optimal solution can use only 4 ADMs, thus $\rho \geq \frac{7}{4}$, a contradiction. Hence, $w(B D G)=2$.

When the third lightpath in the input $y=\mathrm{BAE}$ arrives The situation is as depicted in the right side of the figure. It is clear that $w(y) \neq 3$, since otherwise $\rho \geq \frac{6}{3}>\frac{7}{4}$, a contradiction. Thus $w(y)=1$ or $w(y)=2$.

- case a: $w(y)=1$

Let $z=$ EFKMHG be the next lightpath in the input sequence. Clearly $w(z) \neq$ 1. Hence $w(z)=2$ or $w(z)=3$. If $w(z)=2$, when lightpaths GFEAB, EABDG, BDGFE and EABCDG arrive, we get $w(G F E A B)=3, w(E A B D G)$ $=4, w(B D G F E)=5, w(E A B C D G)=6$, and $\rho=\frac{14}{8}=\frac{7}{4}$, a contradiction. In the case $w(z)=3$ for $u=\mathrm{EABDCHG}$ we have $w(u)=4$, and $\rho \geq \frac{9}{5}>\frac{7}{4}$, a contradiction.

- case b: $w(y)=2$

Let $z=$ BDCHG. Clearly $w(z) \neq 2$. Hence $w(z)=1$ or $w(z)=3$. If $w(z)=1$, when lightpaths EABDG, GFEAB, GKFEAB, and EFGDB arrive, we have $w(E A B D G)=3, w(G F E A B)=4, w(G K F E A B)=5, w(E F G D B)=6$, and $\rho \geq \frac{14}{8}=\frac{7}{4}$, a contradiction. In the case $w(z)=3$, for $u=$ GHMKFEAB we have $w(u)=4$. Then $\rho \geq \frac{9}{5}>\frac{7}{4}$, a contradiction.

\subsection{Ring Topology}

The result in the previous Lemma can be proven, though asymptotically even for ring topologies.

Lemma 4.2 No deterministic on-line algorithm has a competitive ratio better than $7 / 4$, even for the ring topology.

Sketch of Proof. We first give the inutitive ideas behind the adversary. Suppose we divide the ring into four segments $R_{1}, R_{2}, R_{3}$ and $R_{4}$. The adversary first requests lightpaths $R_{1}$ and $R_{3}$. 
- If the on-line algorithm assigns the same color to them, we then request two lightpaths $\left(R_{2}, R_{3}, R_{4}\right)$ and $\left(R_{4}, R_{1}, R_{2}\right)$. The on-line algorithm uses 8 ADMs while the offline algorithm can use 4 ADMs.

- If the on-line algorithm assigns different colors to them, we then request $R_{2}$. If the on-line algorithm assigns a third color to $R_{2}$, we further request $R_{4}$ making the on-line algorithm using at least 7 ADMs and the offline algorithm using 4 ADMs only.

The only problematic case for the adversary is that the on-line algorithm assigns $R_{1}$ and $R_{2}$ with the same color and $R_{3}$ using a different color. In this case, the adversary requests two lightpaths $\left(R_{2}, R_{3}, R_{4}\right)$ and $\left(R_{3}, R_{4}, R_{1}\right)$. Neither of these can share ADMs with existing lightpaths. The on-line algorithm uses 7 ADMs plus $2 \mathrm{ADMs}$ for $R_{3}$ while the offline algorithm uses 4 ADMs plus 2 ADMs for $R_{3}$. The adversary then repeats the process for $k$ times such that the on-line algorithm uses $7 k+2$ ADMs and the offline algorithm uses $4 k+2$ ADMs. This gives a competitive ratio at least $\frac{7}{4}-\epsilon$ for any $\epsilon>0$. The crucial point in repeating the process is to ensure later arrival lightpaths cannot share ADMs with lightpaths in previous iterations. This can be done by careful division of the ring and shifting of the division in every iterations. The details can be found in [SWZ07.

\subsection{Path Topology}

Lemma 4.3 For any $\epsilon>0$, there is no $\left(\frac{3}{2}-\epsilon\right)$-competitive deterministic algorithm for path topology.

Proof. We prove using the following adversary. Let $G$ be a path with $2 k$ nodes $u_{1}, v_{1}, u_{2}, v_{2}, \ldots, u_{k}, v_{k}$ (see Figure 3). Let ALG be any deterministic algorithm. The value of $k$ will be determined later.

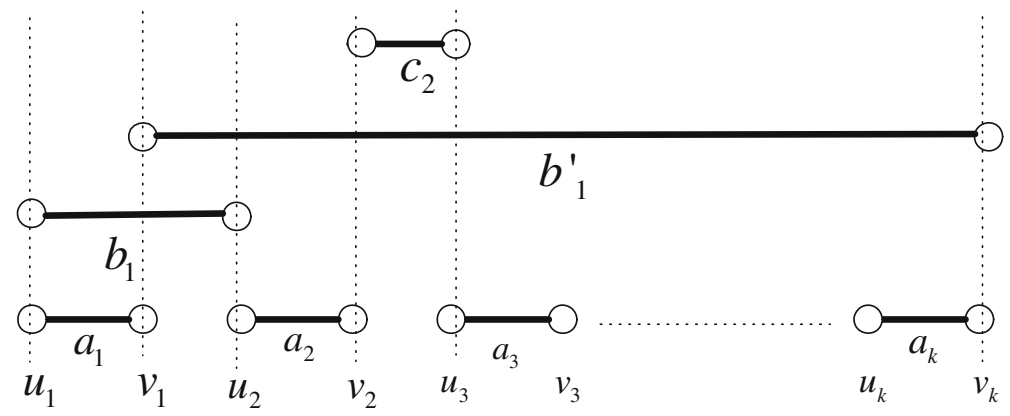

Fig. 3. Proof of Lemma 4.3

The adversary works in two phases. In the first phase the input is $a_{1}, a_{2}, \ldots, a_{k}$ where $\forall i, a_{i}=\left(u_{i}, v_{i}\right)$. In the second phase the input depends on the decisions made by ALG during the first phase. For every $1 \leq i<k$, if $w\left(a_{i}\right)=w\left(a_{i+1}\right)$ then the input contains two paths $b_{i}=\left(u_{1}, u_{i+1}\right)$ and $b_{i}^{\prime}=\left(v_{i}, v_{k}\right)$, otherwise the input contains one path $c_{i}=\left(v_{i}, u_{i+1}\right)$. 
Let $0 \leq x \leq k-1$ be the number of times $w\left(a_{i}\right)=w\left(a_{i+1}\right)$ is satisfied. Then $w\left(a_{i}\right) \neq w\left(a_{i+1}\right)$ is satisfied $k-1-x$ times.

During the first phase the algorithm uses $2 k$ ADMs, one for each node.

For the paths $b_{i}$ and $b_{i}^{\prime}$, let $\lambda=w\left(a_{i}\right)\left(=w\left(a_{i+1}\right)\right) . \lambda$ is not feasible neither for $b_{i}$ nor for $b_{i}^{\prime}$. Then the algorithm assigns other colors to $b_{i}$ and $b_{i}^{\prime}$, and it uses 4 ADMs, for a total of $4 x$ ADMs.

For the path $c_{i}$, let $\lambda=w\left(a_{i}\right)$ and $\lambda^{\prime}=w\left(a_{i+1}\right)(\neq \lambda)$, coloring $c_{i}$ with one of these colors ALG uses one ADM, otherwise it uses 2 ADMs. Therefore for the paths $c_{i}$, ALG uses at least $k-1-x$ ADMs.

Summing up, we get that ALG uses at least $2 k+4 x+(k-1-x)=3(k+x)-1$ ADMs.

On the other hand the following solution is possible. For any consecutive paths $c_{i}, c_{i+1}, \ldots, c_{i+j}$ color such that $w\left(b_{i-1}\right)=w\left(a_{i}\right)=w\left(c_{i}\right)=w\left(a_{i+1}\right)=w\left(c_{i+1}\right)=$ $\ldots=w\left(c_{i+j}\right)=w\left(a_{i+j+1}\right)=w\left(b_{i+j+1}^{\prime}\right)$. This solutions use $2 k+2 x$ ADM's, one $\mathrm{ADM}$ at each $u_{i}, v_{i}, x$ additional ADMs at $u_{1}$, and $x$ additional ADMs at $v_{k}$.

Therefore the competitive ratio of ALG is at least $\frac{3(k+x)-1}{2(k+x)}=\frac{3}{2}-\frac{1}{2(k+x)} \geq$ $\frac{3}{2}-\frac{1}{2 k}$. For any $\epsilon>0$ we can choose $k>\frac{1}{2 \epsilon}$, so that the competitive ratio of $\mathrm{ALG}$ is bigger then $\frac{3}{2}-\epsilon$.

\section{Triangle Topology}

In the previous sections we have shown that algorithm ONLINE-MINADM has an optimal competitive ratio, in general topologies, ring and path topologies. In this section we show an example of topology for which ONLINE-MINADM is not optimal. Note that the proof of Lemma 3.1 implies that ONLINE-MINADM is $\frac{7}{4}$-competitive in the triangle topology. We will show in this section a tight bound of $\frac{5}{3}$ for this topology. Note that the lower bound proof for ring networks requires the ring to be of unbounded size. The proof will not hold for rings of a bounded size. In this section we show that this lower bound does not hold for triangles, and give an optimal algorithm for this topology.

Lemma 5.1 There is no on-line algorithm with competitive ratio $<\frac{5}{3}$ for triangle topology.

Proof. Consider a triangle with edge set $\left\{e_{1}, e_{2}, e_{3}\right\}$. We will use the following adversary.

Release two lightpaths each of length 1 , on edges $e_{1}$ and $e_{2}$. If $w\left(e_{1}\right)=w\left(e_{2}\right)$, then we continue as in Lemma 3.1. namely release two lightpaths of length 2 each $\left\{(e 2-e 3),\left(e_{1}, e_{3}\right\}\right.$, and we get a competitive ratio of $7 / 4>5 / 3$.

Otherwise $w\left(e_{1}\right) \neq w\left(e_{2}\right)$, w.l.o.g. assume $w\left(e_{1}\right)=1, w\left(e_{2}\right)=2$. Release a lightpath on edge $e_{3}$. If $w\left(e_{3}\right) \notin\{1,2\}$ then the competitive ratio is $6 / 3=2>$ $5 / 3$, otherwise w.l.o.g $w\left(e_{3}\right)=1$. In this case we have $w\left(e_{1}\right)=w\left(e_{3}\right)=1$ using 3 ADMs, $w\left(e_{2}\right)=2$ using 2 ADMs, for a total of 5 ADMs. The competitive ratio is $5 / 3$.

For the triangle topology, let us name the three edges in the triangle network $e_{1}, e_{2}$, and $e_{3}$. There are only six types of lightpaths, namely, $\left(e_{1}\right),\left(e_{2}\right),\left(e_{3}\right)$, 
$\left(e_{1}, e_{2}\right),\left(e_{2}, e_{3}\right)$ and $\left(e_{1}, e_{3}\right)$. For any lightpath $p$, we say that $p$ is length $i$ if it contains $i$ edges. There are only length- 1 and length- 2 lightpaths in a triangle topology.

We now present another algorithm ONLINE-TRIANGLE and show that it is 5/3-competitive for triangle topology. Roughly speaking, the algorithm gives highest priority to a pair of length-2 and length-1 lightpaths to share the same color whenever possible. For length-1 lightpaths, we have seen in the lower bound of ONLINE-MINADM in Lemma 3.1 that, if an on-line algorithm always colors two adjacent length-1 lightpaths with the same color, the competitive ratio of the algorithm is at least $\frac{7}{4}$. To overcome this barrier, when a length-1 lightpath, say $p_{i}=\left(e_{1}\right)$, arrives, ONLINE-TRIANGLE does not always color $p_{i}$ with an adjacent length-1 lightpath using the same color. However, if we color three length-1 lightpaths on a cycle each with a different color, this will result in a competitive ratio of 2 . Therefore, if there are two lightpaths $p_{j}=\left(e_{2}\right)$ and $p_{k}=\left(e_{3}\right)$ with different colors, then ONLINE-TRIANGLE should color $p_{i}$ with either of these colors if it is feasible. We formalize this concept by "marking" the three lightpaths to represent they are grouped together and should not be further considered when other length-1 lightpaths arrive.

Formally, the algorithm runs as follows. When a request of lightpath $p_{i}$ with endpoints $u_{i}$ and $v_{i}$ arrives,

1. In case $p_{i}$ is length-2,

- If there exists a length-1 (marked or unmarked) lightpath with color $\lambda$ with endpoints $u_{i}, v_{i}$, and $\lambda$ is feasible for $p_{i}$, then assign $w\left(p_{i}\right)=\lambda$.

- Otherwise, assign $w\left(p_{i}\right)=\lambda^{\prime}$, where $\lambda^{\prime}$ is an unused color.

2. In case $p_{i}$ is length-1,

- If there exists a length-2 lightpath with color $\lambda$ with endpoints $u_{i}, v_{i}$, and $\lambda$ is feasible for $p_{i}$, then assign $w\left(p_{i}\right)=\lambda$.

- Otherwise, if there exists a valid chain of two unmarked length-1 lightpaths with different colors $\lambda_{1}$ and $\lambda_{2}$ with endpoints $u_{i}, v_{i}$, and $\lambda_{1}$ or $\lambda_{2}$ is feasible for $p_{i}$ (w.l.o.g. assume $\lambda_{1}$ is feasible), then assign $w\left(p_{i}\right)=\lambda_{1}$ and mark all three lightpaths involved.

- Otherwise, assign $w\left(p_{i}\right)=\lambda^{\prime}$, where $\lambda^{\prime}$ is an unused color.

For example, suppose $P=\left\{p_{1}, p_{2}, \cdots, p_{7}\right\}$ where $p_{i}$ is, in order, $\left(e_{1}\right),\left(e_{2}\right)$, $\left(e_{3}\right),\left(e_{2}\right),\left(e_{1}\right),\left(e_{3}\right),\left(e_{1}, e_{3}\right)$. Then ONLINE-TRIANGLE will first assign $w\left(p_{1}\right)=$ $\lambda_{1}, w\left(p_{2}\right)=\lambda_{2}, w\left(p_{3}\right)=\lambda_{1}$ and mark all three $p_{1}, p_{2}$ and $p_{3}$. Next, we assign $w\left(p_{4}\right)=\lambda_{3}$ because there is no unmarked lightpath available. We further assign $w\left(p_{5}\right)=\lambda_{4}$ and $w\left(p_{6}\right)=\lambda_{3}$. Finally, we assign $w\left(p_{7}\right)=\lambda_{2}$ because $p_{7}$ and $p_{2}$ form a cycle.

To analyze the performance of ONLINE-TRIANGLE, we first observe how lightpaths are colored in an optimal solution. The proof of the following lemma follows immediately from the definitions.

Lemma 5.2 The optimal solution $S^{*}$ always colors $\left(e_{1}, e_{2}\right)$ and $\left(e_{3}\right)$ with the same color if possible and similarly for the two other symmetric cases. Any 
remaining length-2 lightpath is colored a distinct color. If there are some length1 lightpaths remained after this, cycles of three length-1 lightpaths are colored the same color; followed by chains of two length-1 lightpaths with same color and finally remaining length-1 lightpaths with distinct colors. It can be verified such coloring uses the minimum number of $A D M s$.

We then compare $S$ and $S^{*}$ as follows. We first give a rough idea before formally prove it in Lemma 5.3. Roughly speaking, in $S$, a length-2 lightpath can always share ADM with a length-1 lightpath unless the length-1 lightpath has been marked with the same color with some other length-1 lightpath. In this case, $S^{*}$ also has to use extra ADMs for these this length-1 lightpath, therefore, making $S^{*}$ use a comparable number ADMs as $S$ in total. As mentioned before, ONLINETRIANGLE does not always color adjacent length-1 lightpaths using same color to avoid the $\frac{7}{4}$ lower bound. Furthermore, there is no marked cycle of length-1 lightpaths all with different color; for any marked cycle, $S^{*}$ uses at least 3 ADMs for such cycle while $S$ uses at most 5 , which is indeed the worst case leading to the $\frac{5}{3}$-competitive ratio. Also for the case $S^{*}$ is able to color two length-1 lightpaths with the same color while $S$ has to use two different colors, this only gives a ratio of $\frac{4}{3}$. Precisely, we prove the competitive ratio in the following lemma giving more details.

\section{Lemma 5.3 ONLINE-TRIANGLE is $\frac{5}{3}$-competitive in the triangle topology.}

Sketch of Proof. Consider the solution $S$, the lightpaths can be partitioned into five disjoint sets according to how they are colored. We start with defining the set $A$ whose edges will not be included in later sets and similarly for other sets. Let $A$ be the set of cycles containing a length-1 lightpath and a length-2 lightpath with the same color; $B$ be the set of length-2 lightpaths with distinct color; $C$ be the set of marked cycles containing two same colored length-1 lightpaths and a third different colored one (excluding those later share color with a length-2 lightpath and thus included in $A$ ); $D$ be the set of marked chains containing two same colored length-1 lightpaths (excluding those in $A$ or $C$ ); and $E$ be the set of remaining length-1 lightpaths. In the example given above, $A$ contains $p_{7}$ and $p_{2} ; C$ contains $p_{4}, p_{5}$ and $p_{6} ; D$ contains $p_{1}$ and $p_{3} ; B$ and $E$ are empty.

We denote $|A|,|B|,|C|,|D|$, and $|E|$ by $a, b, c, d$ and $e$, respectively. Note that $\operatorname{cost}(S)=2 a+2 b+5 c+3 d+2 e$.

We consider four cases depending on the set $B$. Case 1: $B$ is empty, in other words, every length-2 lightpath is colored the same color as a length-1 lightpath; this is actually the same as in $S^{*}$. For length-1 lightpaths, by Lemma 5.2, $S^{*}$ colors all possible cycle of 3 lightpaths in the same color using 3 ADMs, then chains of 2 lightpaths with same color using 3 ADMs, and finally 1 lightpath with its own color using 2 ADMs. $S$ needs at most 5, 4 and 2 ADMs for each of the cases, respectively. Therefore, $\frac{S}{S^{*}} \leq \frac{5}{3}$.

Case 2: $B$ contains all three types of length-2 lightpaths. In this case, both $C$ and $E$ must be empty, otherwise, ONLINE-TRIANGLE would have colored some lightpath $p$ in $B$ with the same color as the corresponding lightpath in $C$ or $E$, then $p$ should be in $A$ instead. In this case $S^{*}$ outperform $S$ by grouping 
lightpaths in $B$ with lightpaths in $D$. Even if so, there are still $2 d-b$ length-1 lightpaths left unpaired in $D$. So $S^{*}$ uses at least $2 a+2 b+2 d-b$ ADMs while $S$ uses $2 a+2 b+3 d$ ADMs. Then, $\frac{S}{S^{*}} \leq \frac{3}{2}$.

In Case 3, $B$ contains two types of length-2 lightpaths only; w.l.o.g., assume they are $\left(e_{1}, e_{2}\right)$ and $\left(e_{2}, e_{3}\right)$. In Case $4, B$ contains one type of length-2 lightpaths only; . For these two cases, we can employ a similar argument as in Cases 1 and 2 and show that $\frac{S}{S^{*}} \leq \frac{5}{3}$. The full details can be found in [SWZ07].

\section{Conclusion and Possible Improvements}

In this paper we presented an on-line algorithm with competitive ratio of $\frac{7}{4}$ for any network topology, and proved that no algorithm has a competitive ratio better than $\frac{7}{4}$, even if the topology is a ring. We showed that the same algorithm has a competitive ratio of $\frac{3}{2}$ in path topologies, and that this is also a lower bound for any on-line algorithm on this topology. The lower bound on ring topology does not hold when the ring is of a bounded size; we showed an optimal bound of $\frac{5}{3}$ for the competitive ratio for the triangle topology, using a different algorithm. The analyses of the upper bounds, as well as those for the lower bounds, are all using a variety of proof techniques, which are of interest by their own, and which might prove helpful in future research on the topic.

Our bounds pertain to deterministic on-line algorithms. It may be interesting to explore probabilistic algorithms and obtain similar bounds. Following our study, it might be interesting to determine the exact complexity of the on-line problem for tree topologies, as a function of some parameter of the tree, and of networks (e.g., rings or paths) of bounded size. An important extension is to consider the on-line version of the problem when grooming is allowed; in graphtheoretic terms, this amounts to coloring the paths so that at most $g$ of them are crossing any edge, and where each ADM can serve up to $g$ paths that come from at most two of its adjacent edges (see GRS98, ZM03]). Another direction of extension is to the case where more involved switching functions are under consideration.

\section{References}

[BEY98] Borodin, A., El-Yaniv, R.: Online Computation and Competitive Analysis. Cambridge University Press, Cambridge (1998)

[CFW02] Călinescu, G., Frieder, O., Wan, P.-J.: Minimizing electronic line terminals for automatic ring protection in general wdm optical networks. IEEE Journal of Selected Area on Communications 20(1), 183-189 (2002)

[CW02] Călinescu, G., Wan, P.-J.: Traffic partition in wdm/sonet rings to minimize sonet adms. Journal of Combinatorial Optimization 6(4), 425-453 (2002)

[EL04] Epstein, L., Levin, A.: Better bounds for minimizing sonet adms. In: 2nd Workshop on Approximation and Online Algorithms (September 2004)

[EMZ02] Eilam, T., Moran, S., Zaks, S.: Lightpath arrangement in survivable rings to minimize the switching cost. IEEE Journal of Selected Area on Communications 20(1), 172-182 (2002) 
[FSZ06a] Flammini, M., Shalom, M., Zaks, S.: On minimizing the number of adms tight bounds for an algorithm without preprocessing. In: Erlebach, T. (ed.) CAAN 2006. LNCS, vol. 4235, Springer, Heidelberg (2006)

[FSZ06b] Flammini, M., Shalom, M., Zaks, S.: On minimizing the number of adms in a general topology optical network. In: Dolev, S. (ed.) DISC 2006. LNCS, vol. 4167, pp. 459-473. Springer, Heidelberg (2006)

[GLS98] Gerstel, O., Lin, P., Sasaki, G.: Wavelength assignment in a wdm ring to minimize cost of embedded sonet rings. In: INFOCOM'98. 17th Annual Conference of the IEEE Computer and Communications Societies, pp. 6977. IEEE Computer Society Press, Los Alamitos (1998)

[GRS98] Gerstel, O., Ramaswami, R., Sasaki, G.: Cost effective traffic grooming in wdm rings. In: INFOCOM'98. 7th Annual Conference of the IEEE Computer and Communications Societies. IEEE Computer Society Press, Los Alamitos (1998)

[LV98] Leonardi, S., Vitaletti, A.: Randomized lower bounds for online path coloring. In: 2nd International Workshop on Randomization and Approximation Techniques in Computer Science, pp. 232-247 (1998)

[SWZ07] Shalom, M., Wong, P.W., Zaks, S.: Optimal on-line colorings for minimizing the number of adms in optical networks. In: Technion, Faculty of Computer Science, Technical Report CS-2007-14 (July 2007)

[SZ04] Shalom, M., Zaks, S.: A 10/7 + $\epsilon$ approximation scheme for minimizing the number of adms in sonet rings. In: First Annual International Conference on Broadband Networks, pp. 254-262 (October 2004)

[ZM03] Zhu, K., Mukherjee, B.: A review of traffic grooming in wdm optical networks: Architecture and challenges. Optical Networks Magazine 4(2), 55-64 (2003) 